\title{
Multilayer Perceptron Scheme for Beamforming And Channel Estimation of Massive MIMO
}

\author{
Sneha V.V, Ismayil Siyad C, S.Tamilselvan
}

\begin{abstract}
Massive MIMO being one of the core enabler of $5 G$ network, estimation of channel characteristics in such network plays a vital role. Also the channel is most affected by the phase information rather than the amplitude information. Thus phase estimation plays a very important role in order to ensure error free transmission especially in the case of rapidly changing channels like in massive MIMO. When an accurate transmit beamforming technique is employed to this scheme, it results in maximum user data separation thereby improving the directivity of the signal. The proposed scheme works on two multilayer perceptron (MLP) mechanism model, one for beamforming and other for channel estimation. First MLP model takes the transmitted signal that is passing through the channel to generate the beamforming vectors and these beamforming vectors after prediction are then multiplied with the channel coefficients to enable beamforming. The transmit beamformed signal after passing through the channel and applying appropriate noise, will be received in different directions and these training samples are then given to the second MLP model to predict $D O A$ of the received signal which in turn estimate the channel of massive MIMO. In our proposed scheme, as both beamforming and channel estimation are handled by deep neural network, along with achieving very much better accuracy, mean square error (MSE) and bit error rate (BER) performance, it reduces the number of epochs required for training which results in an efficient learning scheme that is capable of predicting real time rapidly varying channel of massive MIMO.
\end{abstract}

Keywords-MIMO, 5G Communication, Deep learning, Channel estimation, DOA Estimation,Beamforming.

\section{INTRODUCTION}

Traditional approaches of channel estimation, due to their limitation in terms of poor performance and highly complex structures paved way to the integration of deep learning into massive MIMO. In the work mentioned in [1] proposed a time division duplex systems (TDD) Massive

Revised Manuscript Received on July 22, 2019.

Sneha V.V.

PG Scholar, Dept. of ECE, MEA Engineering College,

Perinthalmanna

Ismayil Siyad C.

Assistant Professor, Dept. of ECE, MEA Engineering College,

Perinthalmanna, India

S.Tamilselvan

Associate Professor, Pondicherry Engineering College, Puducherry, India

Email: tamilselvan@pec.edu.in,17pmce08@meaec.edu.in,

ismayilsiyad@meaec.edu.in
MIMO System to acquire CSI in a timely manner by using channel reciprocity. Channel estimation as well as DOA estimation becomes a tedious task when the number of transmitting and receiving antennas are massive. A linear minimum mean square error (LMMSE) algorithm achieves optimal channel estimates with minimal complexity [2] Utilizing the covariance matrix of the imperfect and perfect channel can implement a minimum mean squared error channel estimator with hybrid analog digital estimator which was mentioned in the work [3]. Compressive sensing(CS) for massive MIMO's channel estimation involves base station which is equipped with a very large number of antennas transmitting over a realistic physical channel with finite scattering model. Here a low-rank matrix approximation based on CS is solved through a quadratic semi define programming (SDP) [4]. Classical method of DOA estimation involves delay and sum method in which DOA estimation is done by locating peaks in the spatial spectrum and the other method is capon's minimum variance method [5]. In TLS ESPRIT the sensor array is decomposed to two identical sub arrays which exhibit translational invariance[6]. In the work mentioned in [7] two music algorithms performed in two stages for DOA estimation (both azimuth and elevation angles of impinging sources). Deep learning can analyses network dynamics based on the network parameters Hence it is a powerful tool for analyzing extremely complex wireless networks [8]. Beamforming is one of the widely accepted method in smart antenna technology with an intention of maximizing the directivity of the desired user and there by cancelling the effect of interfering user. Adaptive beamforming algorithms that are working based on the projection of gradient vector shows better performance when compared to the conventional Least mean square(LMS) algorithm [10]. Another work [11] proposed a design that combines both analog and digital beamforming techniques for downlink massive MIMO network. Estimating the real time channel characteristics of massive MIMO is a challenging task as it involves hundreds and even thousands of antenna terminals and each of the channel characteristics are rapidly time varying. In order to tackle this situation a deep learning algorithm is proposed here for channel estimation and beamforming. The main contributions of this work includes:

- Considered two deep learning models, one for transmit beamforming and other for DOA estimation.

- Beamforming is done using MLP and the channel phase is derived from the deep neural network for beamforming. 
- The resulting signal after transmission is obtained in different directions to form the training samples to our MLP model for DOA estimation.

- End to end learning for channel estimation and signal detection.

- Performance of our model using Keras was evaluated for different learning rate and batch size.

Remaining section is organized as follows: Section II depicts the scheme which integrate the massive MIMO with deep learning framework. III section includes Deep learning algorithm for DOA estimation and beamforming. Channel estimation is described in the section IV. Simulation results were given in the $\mathrm{V}$ section and the section VI concludes the work.

\section{SYSTEM MODEL}

A massive MIMO up link system with number of transmitting antennas $\mathrm{T} x=128$ and $\mathrm{Rx}=32$ single antenna users is considered in the proposed scheme. Two deep neural network, one for beamforming and other for DOA estimation is used here. For creating the training samples we have considered Rayleigh and rician based channel model given by (1)

$$
h_{l}=\sum_{p=1}^{m} c_{l i, j} a_{y\left(\theta_{l, j}\right)}
$$

Where $C_{M i}$ denotes the complex gain and $a_{y}\left(\theta_{L_{i j}}\right)$ is the array response. Here "m" resolvable paths are being considered. The array response with the following considerations" $d$ "is the spacing between adjacent antenna terminals," is the wavelength which is given by (2)

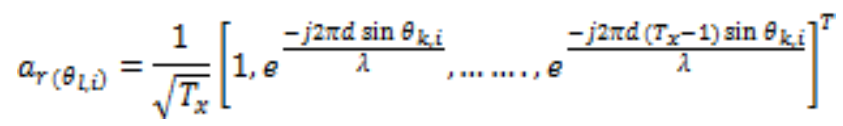

Beamforming is a technique to improve directivity of the signal and to ensure maximum signal separation. This is achieved by multiplying the channel with the inverse phase of channel. This is equivalent to multiplying the signal by beamforming matrix. In this way maximum user separation is ensured. The signal is then corrupted with additive white gaussian noise after passing through the channel. Base station receives the signal and is given by (3)

$y=H x+n$

here" $n$ " denotes the additive white gaussian noise which is used for corrupting the transmitted signal $x ", " H$ " is the channel matrix. Here we have considered test signal of unit magnitude as" x".

\section{BEAMFORMING AND DOA ESTIMATION WITH DEEP LEARNING FRAMEWORK}

A multilayer perceptron mechanism is utilsed for beamforming of the signal. The main purpose of beamforming is to steer the signal in the desired direction of user nullifying the signal in the unintended direction. Multilayer perceptron model with 5 layers are feeded with the input signal in order to predict the beamforming vectors. These beamforming vectors which are the prediction results of first deep neural network is multiplied with the channel coefficients for transmit

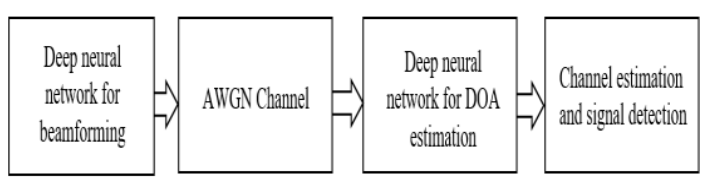

beamforming. The overall process involved in this learning scheme is clearly depicted in Figure.1

\section{Figure 1: Overall process involved in the learning scheme}

In our proposed scheme input is the signals coming from 128 antennas and the output is 32 distinct angles corresponding to each of the recieved signal. Beamforming is done using the deep neural network to predict the beamforming vectors or angle of channel.Most powerful deep learning framework is utilized to its full potential to make the DOA estimation realizable. Sparsity features of massive MIMO is fully utilized here. DOA estimation followed by the signal detection is made achievable purely by the deep learning concept and its powerful representation and recognit: learns through mapping and trainir (1) layer will be triggered by the previous layer neurons. Its output will be the weighed sum of these neurons with their activation function. Output of the network is given by

$\left.Z=f(x, w)=f^{(n-1)}\left(f^{(n-2)}\right) \ldots \cdot f_{x}^{1}\right)$

here" $n$ " denotes the number of layers and" w" denotes the weights. Here we have designed the input layer with dimension equal to the size of training dense layer serving as the input layer

(2) sample. It's a inputed by RELU. Then we have designed 4 hidden layers with $300,256,200,128$ neurons in each layer and each layer functions encoding, corrupting via noise and finally the output layer with 32 neurons which is equal to the number of labels we have used. In the output layer sigmoid function is used as activation. For getting the estimated DOA, loss function is calculated as follows

$l_{2}=\left(E\left\|\theta_{k}-\theta_{k}^{b l}\right\|\right)^{2}$

Mean square error is given by(6)

$M S E_{D O A}=\frac{1}{N} \sum_{j=1}^{N} E\left\|\theta_{k}-\theta_{k}^{N}\right\|^{2}$

Beamforming and DOA estimation framework using deep neural network is shown in Fig.1. The signals applied to the first deep neural network for generating beamforming vectors then these beamforming vectors are given to the channel model, where is it is multiplied with the channel coefficients. Then received signal with different DOAs is obtained. 
Training samples are generated from different channel models. Transmitted signal vector is multiplied with channel coefficients which are kept constant and finally corrupted by AWGN to generate" $y$ ". The received signal" $y$ " in all directions along with their direction forms the training samples. The angles can be set as 4,8,16,32 degrees. In the proposed scheme 32 labels are used. The received signal" $y$ " forms the input and the 32 directions forms the 32 unique labels from 0 to 31 . Once the training is completed the testing is done with random input to predict the DOA.

\section{CHANNEL ESTIMATION}

The channel matrix is composed of two components namely, complex gain information and DOA information. For estimating the complex gain, consider the $j^{\text {th }}$ estimated signal as $y_{j}$ and $x_{j}$ as the jth transmitted signal vector. Now subtracting the effect of noise from the estimated output, it is modified as per [9]

$$
\begin{aligned}
& \overline{y j}=y_{j}^{\mathrm{m}}-n_{j}^{\mathrm{B}} \\
& \overline{y j}=H_{j}^{n} z_{j}^{n}
\end{aligned}
$$

Where $n_{j}$ and $H_{j}^{\mathrm{n}}$ denote the estimated noise and estimated channel matrix respectively. $z_{j}^{\mathrm{R}}$ is the unit test signal.

$\bar{j}_{j}=H_{j}^{n}=A_{y_{a} j}^{n} C_{j}^{n}$

Here $A_{\gamma_{s} j}$ is already obtained during the DOA Estimation. By algebraic transformation Estimate of complex gain can be given as

$C_{j}^{\infty}=\left(A_{r i j}^{n}\right)^{-1-j j}$

Proposed work focused on the channel phase information for estimation rather than its magnitude. In order to reduce complexity, we have taken unity gain for the channel. So the predicted values of DOA is taken to recover the angle for signal detection. Since the complex gain is taken as unity, the recovered angular information gives the detected channel coefficient" H" itself. From equation (3)

$x^{n}=\frac{y^{n}}{H^{n}}$

Depending on the value of $\mathrm{x}$. whether it exceed the threshold or not, it is detected as either 0 or 1 . Finally bit error rate can be found by bit xor operation between transmitted signal and the detected signal. A low BER value ensures the efficient signal detection and channel estimation scheme.

\section{SIMULATION RESULTS}

The most powerful keras which is running with tensorflow backend is used to train our model. Two schemes are considered for analysis. First scheme included deep learning based channel estimation with transmit beamforming and the second scheme deals with deep learning based channel estimation with deep learning enabled transmit beamforming. Performance valuation shows that the second scheme which employed deep learning for both beamforming and channel estimation shows superior performance when comparing to the scheme that includes transmit beamforming without deep learning.

\section{Figure 2: Algorithm for the proposed scheme}

Here we have considered 128 transmitting antennas

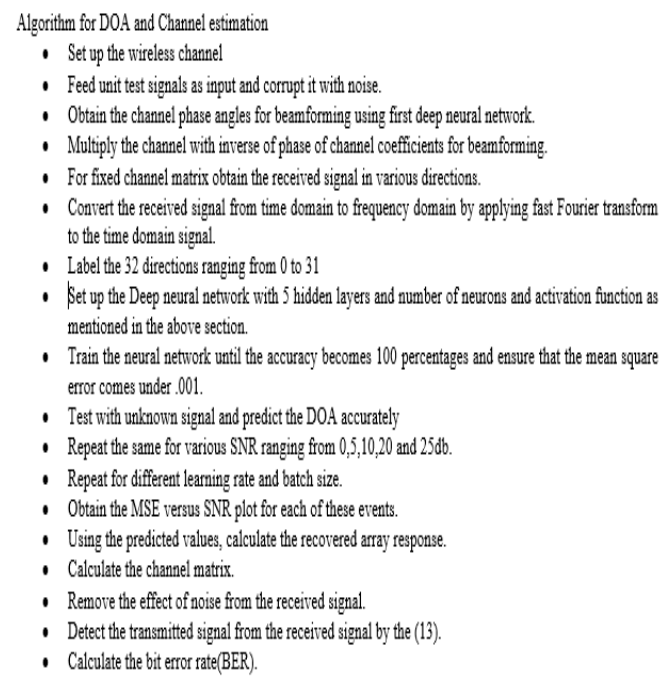

and 32 single antenna users in generating the training samples with spacing between adjacent antenna terminals chosen to be $\mathrm{d}=\lambda / 2$ and the DOA is randomly distributed in. Figure 3. depicts the BER variation as a function of epoch. The whole dataset passed through the neural network in both forward and backward for one time is said to constitute one epoch. It is clear that higher the number of epochs, lower will be the error rate. For a few epochs ranging from 5 to nearly 10 BER decreases and finally settles to value 0 for higher epochs. When comparing with the previous deep learning scheme mentioned in [9] in our work BER drops to 0 near to 15 epochs, where as in the previous work it is achieved only near to 25 epochs. This is because in the proposed scheme a high directivity signal is used for training the deep neural network in frequency domain. Also here beamforming is done using the MLP model. 


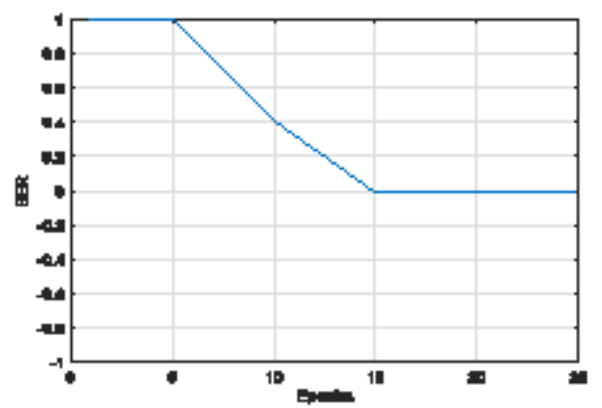

Figure 3: BER variation for different epoch

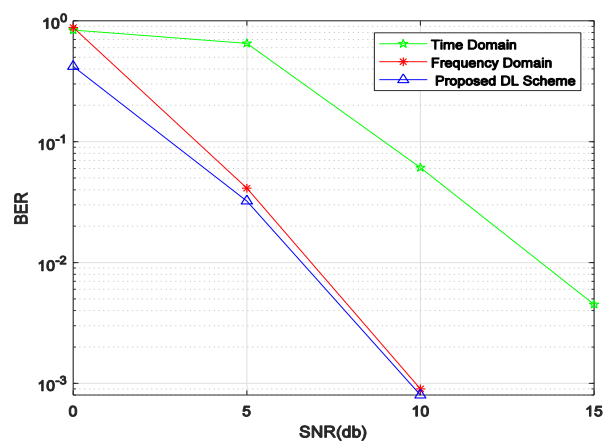

Figure 4: BER comparision for time domain learning, frequency domain learning and deep learning based transmit beamforming scheme.

Figure. 4 depicts BER performance of time domain signal, DL Scheme for DOA and DL Scheme for DOA+Beamforming. Here the BER performance of time domain signal is lagging when comparing to the two other method we have introduced here.

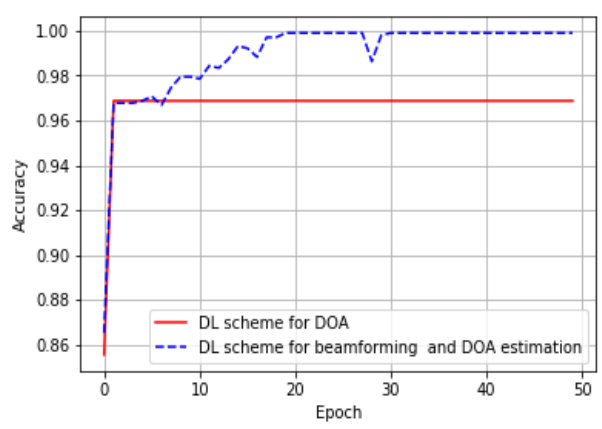

Figure 5: Accuracy comparison DL scheme for DOA estimation and DL based DOA+transmit beamforming scheme

Figure. 5 compares the accuracy obtained from DL Scheme for DOA and DL Scheme for DOA+Beamforming. It is clear that the accuracy plot obtained from the second scheme is much better than the first one, this is due to the deep neural network that we have introduced to the beamforming technique. As beamforming is done using prediction it is much

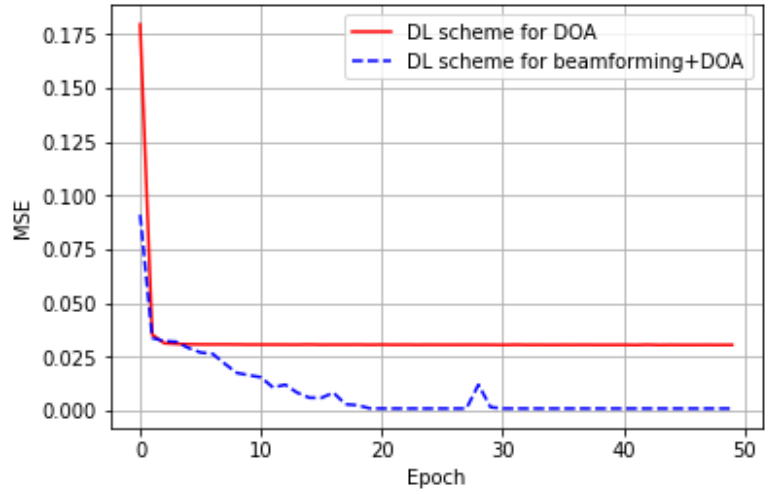

accurate and time saving when comparing to the normal transmit beamforming.

Figure 6: MSE comparison DL scheme for DOA estimation and DL based DOA+transmit beamforming scheme

Figure 6. compares the MSE obtained from DL Scheme for DOA and DL Scheme for DOA+Beamforming. We could notice a significant improvement in MSE performance when comparing to previous schemes [9]. Also when comparing the scheme which utilized a single neural network for DOA Estimation and the other scheme which utilized two neural network, one for beamforming and the other for DOA estimation, second method shows a significant improvement in the MSE value. Here also we could notice that MSE of proposed scheme where DOA+Beamforming utilize Deep learning for both beamforming as well as DOA estimation, drops to very small value in the earlier iterations itself. But this is not the case with the scheme where normal transmit beamforming is used, when looking at the transmit beamforming scheme without deep learning it is clear that MSE is not rapidly reducing, in the later scheme it settles to

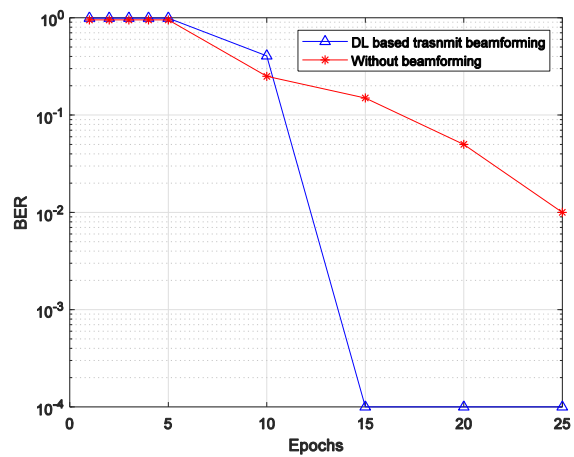

the final value of 0 in around 10 epochs itself.

Figure 7: BER comparison DL scheme for channel estimation and DL based DOA+transmit beamforming scheme

Figure. 7 gives comparision plot of bit error rate for the proposed scheme with deeplearning enabled beamforming and the channel estimation scheme without beamforming proposed in [9]. In proposed scheme the bit error rate starts reducing at 10 epochs and suddenly goes to a minimal value at 15 epoch itself. In case of the work mentioned in [9] BER is 
not decreasing as our scheme and also the error rate is high even at 25 epochs. This clearly shows that our scheme gives a better BER performance for the channel estimation than the previous scheme at a reduced number of iterations itself.

\section{CONCLUSION}

A dual deep neural network implementation is considered in this work which perform beamforming and the channel estimation of massive MIMO in an efficient manner. This learning scheme includes two deep neural network which work cooperatively, one to determine the beamforming vector and other to perform DOA estimation. We have shown that the performance of our deep neural network that works on multilayer perceptron mechanism is highly efficient in real time prediction of channel which is very much applicable to massive MIMO networks. Also when employing this dual network, we could get a considerable reduction in the operation when comparing to scheme that employ a single neural network. Our intention was to achieve the better performance than the state of art techniques with least data and minimal iterations which results in reduced operation time. Also accurate predictions of DOA is obtained due to the deep learning based beamforming technique applied at the transmitter side. The capability of DNN integrated into massive MIMO can bring reduction in computational complexity and can estimate varying channel conditions. Such a system which can estimate the channel parameters even if its nature is unknown and predict the DOA accurately can yield better performance. Along with this optimized channel estimation technique, frequency domain beamforming employed made it possible to reduce the MSE, BER and improve the accuracy which resulted in an optimized communication scheme.

\section{REFERENCES}

[1] Neumann, David, Michael Joham, and Wolfgang Utschick. "Channel estimation in massive MIMO systems." arXiv preprint arXiv:1503.08691 (2015).

[2] Zaib, Alam, et al. "Distributed channel estimation and pilot contamination analysis for massive MIMO-OFDM systems." IEEE Transactions on Communications 64.11 (2016): 4607-4621.

[3] Bogale, Tadilo Endeshaw, Long Bao Le, and Xianbin Wang. "Hybrid analog-digital channel estimation and beamforming: Training-throughput tradeoff." IEEE Transactions on Communications 63.12 (2015): 5235- 5249.

[4] Gao, Zhen, et al. "Spatially common sparsity based adaptive channel estimation and feedback for FDD massive MIMO.'IEEE Transactionson Signal Processing 63.23 (2015): 6169-6183.

[5] Weber, Raymond J., and Yikun Huang. "Analysis for Capon and MUSIC DOA estimation algorithms." 2009 IEEE Antennas and Propagation Society International Symposium. IEEE, 2009.

[6] Dheringe, N. A., and B. N. Bansode. "Performance evaluation and analysis of direction of arrival estimation using MUSIC, TLS ESPRIT and Pro ESPRIT algorithms.” Perform. Eval 4.6 (2015): 4948-4958.

[7] Yang, Kai-Yu, Jwo-Yuh Wu, and Wen-Hsuan Li. "A low-complexity direction-of-arrival estimation algorithm for full-dimension massive MIMO systems.” 2014 IEEE International Conference on Communication Systems. IEEE, 2014.

[8] Mao, Qian, Fei Hu, and Qi Hao. ’Deep learning for intelligent wireless networks: A comprehensive survey." IEEE Communications Surveys and Tutorials 20.4 (2018): 2595-2621.

[9] Huang, Hongji, et al. "Deep learning for super-resolution channel estimation and DOA estimation based massive MIMO system." IEEE Transactions on Vehicular Technology 67.9 (2018): 8549-8560.
[10] Zhou, Yuanjian, and Xiaohui Yang. "A novel adaptive beamforming algorithm for smart antenna system." 2016 12th International Conference on Computational Intelligence and Security (CIS). IEEE, 2016.

[11] Bogale, Tadilo Endeshaw, and Long Bao Le. "Beamforming for multiuser massive MIMO systems: Digital versus hybrid analog-digital." 2014 IEEE Global Communications Conference. IEEE, 2014. 